Krzysztof Bolibok · Kazimierz Goebel · W. A. Kirk

\title{
Remarks on the stability of the fixed point property for nonexpansive mappings
}

Received: 11 May 2012 / Accepted: 14 September 2012 / Published online: 6 October 2012

(C) The Author(s) 2012. This article is published with open access at Springerlink.com

\begin{abstract}
It is shown that in many instances the fixed point property for nonexpansive mappings actually implies the fixed point property for a strictly larger family of mappings. This paper is largely expository, but some of the observations are not readily available, and some appear here for the first time. Several related open questions in are discussed. The emphasis is on accessible problems, especially those that require little background. The problems themselves have been given little thought and may be trivial or difficult.
\end{abstract}

\section{Mathematics Subject Classification $47 \mathrm{H} 10$}

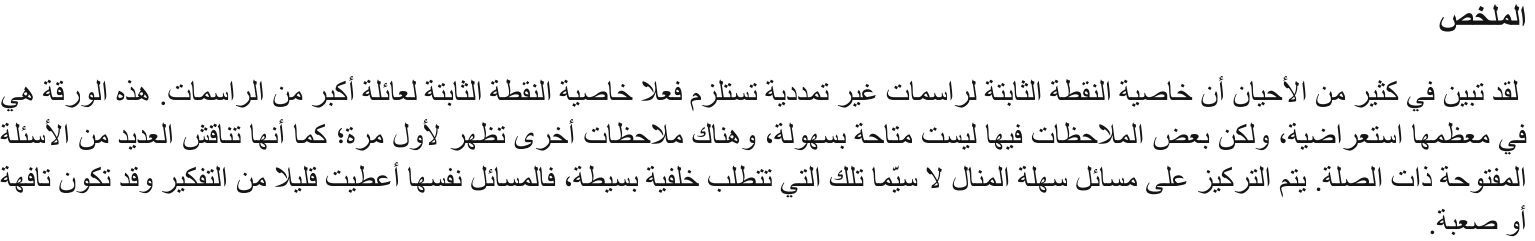

\section{Introduction}

Elementary problems are not necessarily easy problems. Often quite the opposite is true. Basic problems which are easy to formulate can inspire curiosity, but often there are there few tools available to suggest a viable approach. Also, as always, there is the real possibility that only a counterexample will solve the problem, and it seems that often people prefer proving theorems over looking for examples. However, as we shall see, examples are often helpful to a full understanding of the theory.

The central theme of these remarks is to illustrate the following fact: the fixed point property for nonexpansive mappings often implies the fixed point property for a strictly broader class of mappings. Examples help reveal the limitations.

K. Bolibok $\cdot$ K. Goebel

Institute of Mathematics, Maria Curie-Skłodowska University, 20031 Lublin, Poland

E-mail: bolibok@hektor.umcs.lublin.pl

K. Goebel

E-mail: goebel@hektor.umcs.lublin.pl

W. A. Kirk ( $\varangle)$

Department of Mathematics, University of Iowa, Iowa, IA 52242, USA

E-mail: william-kirk@uiowa.edu 


\section{The fixed point property for nonexpansive mappings}

The study of nonexpansive mappings originated in a Banach space framework. Let $K$ be a closed convex subset of a Banach space $(X,\|\cdot\|)$. A mapping $T: K \rightarrow K$ is said to be nonexpansive if

$$
\|T x-T y\| \leq\|x-y\| \quad \text { for all } x, y \in K .
$$

We say that $K$ has the fixed point property (fpp for short) if every nonexpansive $T: K \rightarrow K$ has at least one fixed point. We say that the space $X$ has the $F P P$ if every bounded closed convex subset $K$ of $X$ has the fpp. In studying the fpp in Banach spaces it is typical to assume the set $K$ is weakly compact, although it is known that this condition alone is neither necessary nor sufficient. For the FPP one typically assumes that the space is reflexive. However, to this day, it remains unknown whether reflexivity (or even super-reflexivity) alone is sufficient for the FPP. For much of what is currently known about the fixed point theory for nonexpansive mappings we refer to the Handbook [35], and especially in the survey articles [25] and [17].

Three papers (Browder [6], Göhde [30], and Kirk [34]), published by coincidence in 1965, provided the foundation for the theory. In [6] and [30], it was shown that a uniformly convex Banach space has the FPP; in [34], it was shown that the same conclusion follows under the weaker assumptions that $X$ is reflexive and the bounded convex subsets of $X$ posses a geometric condition called 'normal structure'. (We recall that a convex subset $K$ of $X$ has normal structure if every bounded convex subset $H$ of $K$ which contains more than one point contains a nondiametral point, that is, a point $y_{0}$ such that

$$
\left.\sup \left\{\left\|y_{0}-x\right\|: x \in H\right\}<\sup \{\|y-x\|: x, y \in H\} .\right)
$$

It was discovered later that if $X$ is uniformly convex, then in fact the bounded closed convex subsets of $X$ have the fixed point property for a broader class of mappings. Let $K$ be a bounded closed convex subset of a uniformly convex space; suppose $T: K \rightarrow K$ is uniformly lipschitzian in the sense that

$$
\left\|T^{n} x-T^{n} y\right\| \leq k\|x-y\|
$$

for all $x, y \in K$ and $n=1,2, \ldots$ It was shown in [22] that if $k>1$ is sufficiently near 1 (how near depends on the modulus of convexity), then $T$ always has a fixed point. This prompted further the study of 'stability' of the fpp and it is the starting point of the following remarks.

\section{The Lifšic constant and uniformly lipschitzian mappings}

Let $(M, \rho)$ be a metric space. A mapping $T: M \rightarrow M$ is said to be lipschitzian if there exists $k>0$ such that for each $x, y \in M$,

$$
\rho(T x, T y) \leq k \rho(x, y) .
$$

We will use $k(T)$ to denote the smallest number for which (1) holds. The mapping $T: M \rightarrow M$ is said to be $k$-uniformly lipschitzian if for each $x, y \in M$ and all $n \in \mathbb{N}$,

$$
\rho\left(T^{n} x, T^{n} y\right) \leq k \rho(x, y) .
$$

The smallest number for which (2) holds is called the uniform Lipschitz constant of $T$ and denoted $k_{u}(T)$. Obviously for any nonexpansive mapping $T, k_{u}(T) \leq 1$.

The result of [22] was subsequently refined in [27] using the following concept due to Goebel:

Definition 3.1 [18] The characteristic of convexity of a Banach space $X$ is defined to be the number

$$
\varepsilon_{0}(X):=\sup \left\{\varepsilon \in[0,2]: \delta_{X}(\varepsilon)=0\right\}
$$

where $\delta_{X}$ is the usual modulus of convexity of $X$

$$
\delta(\varepsilon)=\inf \left\{1-\left\|\frac{x+y}{2}\right\|:\|x\| \leq 1,\|y\| \leq 1,\|x-y\| \geq \varepsilon\right\} .
$$


Observe that if $\gamma$ satisfies

$$
\gamma\left(1-\delta_{X}(1 / \gamma)\right)=1
$$

then $\gamma \geq 1$. Moreover, $\gamma>1 \Leftrightarrow \varepsilon_{0}(X)<1$, in which case $1<\gamma<1 / \varepsilon_{0}(X)$.

Theorem 3.2 [22,27] Let $X$ be a Banach space with $\varepsilon_{0}(X)<1$ and let $\gamma>1$ satisfy $\gamma\left(1-\delta_{X}(1 / \gamma)\right)=1$. If $K$ is a nonempty closed, bounded convex subset of a Banach space, and if for some $k<\gamma, T: K \rightarrow K$ satisfies

$$
\left\|T^{n} x-T^{n} y\right\| \leq k\|x-y\|
$$

for $n=1,2, \ldots$, then $T$ has a fixed point.

Theorem 3.2 was subsequently extended to a purely metric setting by Lifšic [38]. We turn now to this extension. Let $(M, \rho)$ be a complete metric space. The balls in $M$ are said to be $c$-regular if for each $k<c$ there exist $\mu, \alpha \in(0,1)$ such that $\forall x, y \in M$ and $r>0$ there exists $z \in M$ such that

$$
B(x ;(1+\mu) r) \cap B(y ; k(1+\mu) r) \subset B(z ; \alpha r) .
$$

The balls in $M$ are always 1-regular. To see this, suppose $k<1$. Then it is possible to choose $\mu$ so near 0 that

$$
k(1+\mu):=\alpha<1
$$

in which case $\forall x, y \in M$ and $r>0$,

$$
B(x ;(1+\mu) r) \cap B(y ; k(1+\mu) r) \subset B(y ; \alpha r) .
$$

The Lifsic constant of $M$ is the number

$$
\kappa(M)=\sup \{c \geq 1: \text { the balls in } M \text { are } c \text {-regular }\} .
$$

Theorem 3.3 (Lifšic) If $(M, \rho)$ is a bounded complete metric space, and if for some $k<\kappa(M), T: M \rightarrow M$ satisfies

$$
\rho\left(T^{n} x, T^{n} y\right) \leq k \rho(x, y)
$$

for $n=1,2, \ldots$, then $T$ has a fixed point.

For most metric spaces $M, \kappa(M)=1$ and for such spaces Lifšic's theorem is equivalent to the Banach Contraction Principle. However, as we observe below there are spaces for which $\kappa(M)>1$.

Now let $X$ be a Banach space. We define the uniform Lifšic constant, $\kappa_{0}(X)$, of $X$ as follows:

$$
\kappa_{0}(X)=\sup \left\{c \geq 1: \begin{array}{l}
\exists \alpha<1 \text { such that } \forall x,\|x\| \leq 1, \exists \lambda \in[0,1] \\
\text { such that } B(0 ; 1) \cap B(x ; c) \subset B(\lambda x ; \alpha)
\end{array}\right\} .
$$

infimum of $\kappa(C)$ where $C$ ranges over all nonempty bounded closed convex subsets of $X$. Lifšic proved that $\kappa_{0}(H) \geq \sqrt{2}$ if $H$ is a Hilbert space, and this estimate is sharp. It is noted in [27] that in Hilbert space $\gamma=\sqrt{5} / 2$ is the solution to $\gamma\left(1-\delta_{X}(1 / \gamma)\right)=1$. Therefore, for a Hilbert space Lifšic's estimate on $k$ is better than that given in Theorem 3.2.

The next fact shows that while Lifšic's result sometimes gives a better estimate for $k$, qualitatively Theorems 3.2 and 3.3 are equivalent.

Theorem 3.4 [14] Let $X$ be a Banach space. Then $\varepsilon_{0}(X)<1$ if and only if $\kappa_{0}(X)>1$. 
Mappings satisfying (3) are said to be $k$-uniformly lipschitzian. Such mappings are also characterized by the following observation: recall that two metrics $\rho$ and $\mu$ on a space $M$ are said to be uniformly equivalent if there exist constants $b \geq a>0$ such that

$$
a \mu(x, y) \leq \rho(x, y) \leq b \mu(x, y)
$$

for all $x, y \in M$. If $\mu$ satisfies (4) and if $T: M \rightarrow M$ is $\mu$-nonexpansive-thus $\mu(T x, T y) \leq \mu(x, y)$ for all $x, y \in M$ - then $T$ satisfies (3) with $k=\frac{b}{a}$. On the other hand, if $T$ satisfies (3), then $T$ is $\mu$-nonexpansive with respect to the metric

$$
\mu(x, y)=\sup \left\{\rho(x, y), \rho(T x, T y), \rho\left(T^{2} x, T^{2} y\right), \ldots\right\} \quad x, y \in M .
$$

Clearly, the two metrics $\rho$ and $\mu$ are equivalent: $\rho(x, y) \leq \mu(x, y) \leq k \rho(x, y)$ for all $x, y \in M$. Thus we have the following kind of stability. Once it is known that a metric space $(M, \rho)$ has the fpp for nonexpansive mappings and that $\kappa(M)>1$, then all metric spaces $(M, \mu)$ satisfying (4) for $\frac{b}{a}<\kappa(M)$ also have the fpp for nonexpansive mappings.

The following remark follows from an examination of the proof of Lifšic's Theorem (see, e.g., [24, p. 172]).

Remark 3.5 Lifšic's Theorem actually holds under weaker assumptions. It is enough to assume that $M$ is a complete metric space and that $T: M \rightarrow M$ has bounded orbits and satisfies

$$
\rho\left(T^{n} x, T^{n} y\right) \leq k_{n} \rho(x, y)
$$

for all $x, y \in M$ with $\lim \sup _{n \rightarrow \infty} k_{n}<\kappa(M)$.

Remark 3.6 See [33] for an extension of Lifšic's Theorem to multivalued mappings.

It is not known if $k<\kappa_{0}(X)$ is the best estimate for the fixed point property for $k$-uniformly lipschitzian mappings defined on closed bounded convex subsets of $X$. Thus it is natural to define the constant

$$
\gamma_{0}(X)=\sup \left\{k: \begin{array}{l}
\text { any closed bounded convex subset } K \subset X \\
\text { has the fpp for } k \text {-uniformly lipschitzian mappings }
\end{array}\right\} .
$$

Clearly for any Banach space $X$,

$$
\gamma_{0}(X) \geq \kappa_{0}(X)
$$

Exact values for $\kappa_{0}(X)$ are difficult to find, even for standard uniformly convex spaces. As we have seen, for a Hilbert space $H, \kappa_{0}(H)=\sqrt{2}$, so $\gamma_{0}(H) \geq \sqrt{2}$. An estimate for $\gamma_{0}(H)$ from above is also known. There is an example, due to Baillon ([2]; also see [24, p. 174]) of a fixed-point free $k$-uniformly lipschitzian mapping $T$ defined on the positive part of the unit sphere of $\ell^{2}$ for $k=\pi / 2$. This shows that

$$
\sqrt{2} \leq \gamma_{0}(H) \leq \frac{\pi}{2} .
$$

There are other estimates for $\gamma_{0}(X)$ which are independent of and, in some instances, better than $\kappa_{0}(X)$. Recall that for any bounded closed convex set $K \subset X$, the Chebyshev radius of $K$ is the number

$$
r(K)=\inf _{x \in K}\{\sup \{\|x-y\|: y \in K\}\}
$$

Obviously, $r(K) \leq \operatorname{diam}(K)$. The normal structure coefficient $N(X)$ is defined to be

$$
\inf \left\{\frac{\operatorname{diam}(K)}{r(K)}: K \subset X \text { is bounded, convex, with diam }(K)>0\right\} .
$$

All the spaces with $N(X)>1$ are said to have uniform normal structure. Among such spaces are the uniformly convex spaces, and spaces $X$ for which $\varepsilon_{0}(X)<1$. In the latter case,

$$
N(X) \geq \frac{1}{1-\delta(1)}>1
$$


However, it may happen that the first inequality is sharp. This happens in the case of a Hilbert space $H$ where

$$
\kappa(H)=N(H)=\sqrt{2}>\left(1-\delta_{H}(1)\right)=\frac{\sqrt{5}}{2} .
$$

The first result using $N(X)$ to evaluate $\gamma_{0}(X)$ is due to Casini and Maluta [11] who proved that for any Banach space $X$,

$$
\gamma_{0}(X) \geq \sqrt{N(X)}
$$

This raises the following question: which estimate (5) or (6) is better for a given space, especially since there are spaces $X$ for which $N(X)>\kappa_{0}(X)$. An example of such a space is the space $X=\mathbb{R} \times H$ where $H$ is a Hilbert space and the norm is taken to be $\|(t, x)\|_{X}=\max \{|t|,\|x\|\}$ for $(t, x) \in \mathbb{R} \times H$. For $(0,0),(0,1) \in X$ the intersection

$$
B((0,0) ; 1) \cap B((0,1) ; 1)
$$

cannot be covered by a ball of diameter less than 1 . This shows that $\kappa_{0}(X)=1$ and $N(X) \geq 1$. On the other hand, the normal structure coefficient of the product of two spaces is infimum of their respective normal structure coefficients (see [3] ). Thus the normal structure coefficient of $X$ is $\sqrt{2}$.

\section{Renorming}

Another approach to stability is connected with renormings of Banach spaces. Recall that two norms $\|\cdot\|,\|\cdot\|^{0}$ on a Banach space $X$ are equivalent if they generate uniformly equivalent metrics. Thus $\|\cdot\|$ and $\|\cdot\|^{0}$ are equivalent if there exist constants $a>0, b>0$ such that for all $x \in X$,

$$
a\|x\|^{0} \leq\|x\| \leq b\|x\|^{0} .
$$

Obviously, any mapping that is nonexpansive with respect to one of these norms is $k$-uniformly lipschitzian with respect to the other, with $k=\frac{b}{a}$.

Now suppose $(X,\|\cdot\|)$ has the FPP. Restricting our considerations only to metrics generated by norms equivalent to $\|\cdot\|$, we can introduce the stability constant, $\gamma_{\mathcal{N}}(X)$, of $X$ as follows:

$$
\gamma_{\mathcal{N}}(X)=\sup \left\{\gamma: \begin{array}{l}
\text { any Banach space }\left(X,\|\cdot\|^{0}\right), \text { with }\|\cdot\|^{0} \text { satisfying } \\
(7), \text { such that } k=\frac{b}{a}<\gamma \text { has the FPP. }
\end{array}\right\}
$$

Obviously,

$$
\gamma_{\mathcal{N}}(X) \geq \gamma_{0}(X)
$$

It is known that for some spaces this inequality is strict. The most well-known result is due to Pei-Kee Lin, who showed in [39] that for Hilbert space $H$

$$
\gamma_{\mathcal{N}}(H) \geq \sqrt{\frac{5+\sqrt{13}}{2}} \approx 2.07>\frac{\pi}{2} \geq \gamma_{0}(H) .
$$

Subsequently, this result was improved by Mazcuñán-Navarro, [42] who obtained the estimate

$$
\gamma_{\mathcal{N}}(H) \geq \sqrt{\frac{5+\sqrt{17}}{2}} \approx 2.135
$$

As far as we know, until now the question of whether a bounded set $K$ in a Banach space may have the fpp, yet be completely unstable relative to $k$-uniformly lipschitzian mappings has not been addressed. Specifically, 
Problem 4.1 Does there exist a convex, closed and bounded set $K$ in a Banach space such that all nonexpansive mappings $T: K \rightarrow K$ have fixed points, but for any $\varepsilon>0$, there exists a uniformly lipschitzian fixed point free mapping $G: C \rightarrow C$ satisfying

$$
\left\|G^{n} x-G^{n} y\right\| \leq k\|x-y\|
$$

for all $x, y \in K$ and $n \in \mathbb{N}$, with $k<1+\varepsilon$ ?

There seem to be no obvious examples. However, a remarkable space discovered by Pei-Kee Lin provides an affirmative answer. It is well known that the space $l^{1}$ of absolutely summable sequences $x=\left(x_{k}\right)$ with its classical norm $\|x\|=\sum_{k=1}^{\infty}\left|x_{k}\right|$ lacks the FPP. Indeed, the positive part of the unit sphere

$$
S^{+}=\left\{x=\left(x_{k}\right):\|x\|=1 \text { and } x_{k} \geq 0 \text { for } k=1,2, \ldots\right\}
$$

is invariant under the shift operator

$$
V x=V\left(x_{1}, x_{2}, x_{3}, \ldots\right)=\left(0, x_{1}, x_{2}, x_{3}, \ldots\right),
$$

and clearly this mapping is nonexpansive (in fact an isometry) but fixed point free.

It therefore came as a surprise to many when Pei-Kee Lin [40] showed that $l^{1}$ can be given a new equivalent norm $\|\cdot\|^{0}$ for which $\left(l^{1},\|\cdot\|^{0}\right)$ has the FPP. Lin's norm is given by

$$
\|x\|^{0}=\max \left\{\gamma_{n} \sum_{k=n}^{\infty}\left|x_{k}\right|: n=1,2, \ldots\right\},
$$

where $\gamma_{n}=\frac{8^{n}}{8^{n}+1}$.

In connection with this, Dowling et al. [13] have shown that if $X$ is a Banach space containing an isometric copy of $l^{1}$, and if $\varepsilon>0$, then there exists a closed convex bounded subset $C$ of $X$ and a fixed point free uniformly lipschitzian mapping $T: C \rightarrow C$ with uniform Lipschitz constant less than $1+\varepsilon$. Consequently, $l^{1}$ cannot be renormed to have the fixed point property for uniformly lipschitzian mappings.

Example The shift operator $V$ is not nonexpansive with respect to Lin's norm $\|\cdot\|^{0}$. It is easy to check that with respect to $\|\cdot\|^{0}$ we have

$$
k(V)=\frac{\gamma_{2}}{\gamma_{1}}, k\left(V^{2}\right)=\frac{\gamma_{3}}{\gamma_{1}}, k\left(V^{3}\right)=\frac{\gamma_{4}}{\gamma_{1}}
$$

and in general

$$
k\left(V^{k}\right)=\frac{\gamma_{k+1}}{\gamma_{1}}, k=1,2,3, \ldots
$$

This implies $k_{u}(V)=\frac{1}{\gamma_{1}}$.

In view of the observations of the preceding section, since $V$ is uniformly lipschitzian with respect to $\|\cdot\|^{0}$ it is nonexpansive with respect to the norm $\|\cdot\|^{00}$ defined by

$$
\|x\|^{00}=\sup \left\{\left\|V^{k} x\right\|^{0}: k=0,1,2, \ldots\right\}
$$

but it is easy to check that this is just the classical norm $\|\cdot\|$ on $l^{1}$, and

$$
\gamma_{1}\|x\| \leq\|x\|^{0} \leq\|x\|^{00}=\|x\|
$$

for all $x \in l^{1}$.

Similar facts are valid for the sequence of mappings $V_{p}, p=1,2,3, \ldots$ defined by

$$
V_{p} x=V\left(x_{1}, x_{2}, \ldots\right)=\left(x_{1}, x_{2}, \ldots, x_{p}, 0, x_{p+1}, x_{p+2}, \ldots\right)
$$


For any $k \geq 1$

$$
V_{p}^{k} x=V_{p}^{k}\left(x_{1}, x_{2}, \ldots\right)=(x_{1}, x_{2}, \ldots, x_{p}, \overbrace{0,0, \ldots, 0}^{k \text {-times }}, x_{p+1}, x_{p+2}, \ldots) .
$$

Now, as above, we have

$$
k\left(V_{p}^{k}\right)=\frac{\gamma_{p+k}}{\gamma_{p}} \text { and } k_{u}\left(V_{p}\right)=\frac{1}{\gamma_{p}}
$$

Thus all the mappings $V_{p}$ are uniformly lipschitzian, with

$$
\lim _{p \rightarrow \infty} k_{u}\left(V_{p}\right)=\lim _{p \rightarrow \infty} \frac{1}{\gamma_{p}}=\lim _{p \rightarrow \infty}\left(1+\frac{1}{8^{p}}\right)=1 .
$$

As above for the straight shift $V$, each $V_{p}$ is nonexpansive relative to the norm

$$
\|x\|_{p}^{00}=\sup \left\{\left\|V_{p}^{k} x\right\|^{0}: k=0,1,2, \ldots\right\}
$$

for $x \in l^{1}$. This norm coincides with the classical norm $\|\cdot\|$ on $l^{1}$, for all $x$ with $x_{1}=x_{2}=\cdots=x_{p}=0$ and satisfies

$$
\gamma_{p}\|x\| \leq\|x\|^{0} \leq\|x\|^{00} \leq\|x\|,
$$

for $x \in l^{i}$.

Now consider the closed convex subset $K$ of $S^{+}$defined by

$$
K=\left\{x=\left(x_{1}, x_{2}, x_{3}, \ldots\right) \in S^{+}: \sum_{i=k}^{\infty} x_{k} \geq \frac{1}{2^{k-1}}\right\} .
$$

The set $K$ is nonempty, and for each $p=1,2,3, \ldots, V_{p}: K \rightarrow K$. Moreover, each $V_{p}$ is fixed point free, because $V_{p} x=x$ implies

$$
\left(x_{1}, x_{2}, \ldots, x_{p}, x_{p+1}, \ldots\right)=\left(x_{1}, x_{2}, \ldots, x_{p}, 0, x_{p+1}, \ldots\right)
$$

and this is only possible if

$$
x_{p+1}=x_{p+2}=x_{p+3}=\cdots=0
$$

However, this contradicts

$$
\sum_{i=p+1}^{\infty} x_{k} \geq \frac{1}{2^{p}}
$$

Therefore, the FPP for $X=\left(l^{1},\|\cdot\|^{0}\right)$ is completely unstable, and

$$
\gamma_{\mathcal{N}}(X)=\gamma_{0}(X)=1 .
$$

We remark that very recently in [31] the authors have obtained new families of renormings of $l^{1}$ satisfying FPP. They also show that the property of failing FPP is not stable for any norm $p(\cdot)$ of $l^{1}$ satisfying $\lim \sup _{n} p\left(x+x_{n}\right)=p(x)+\lim \sup _{n}\left(x_{n}\right)$ for every $w^{*}$-null sequence $\left(x_{n}\right)$ and $x$ in $l^{1}$. 


\section{Mean nonexpansive mappings}

First we observe that if $K$ is a bounded closed convex subset of a Banach space and $T: K \rightarrow K$ is nonexpansive, then a standard argument shows that $T$ always has "approximate fixed points" in the sense that

$$
\inf \{\|x-T x\|: x \in K\}=0 .
$$

One can then ask whether this fact extends to the more general class of mappings satisfying

$$
\min \left\{\|T x-T y\|,\left\|T^{2} x-T^{2} y\right\|\right\} \leq\|x-y\|
$$

for all $x, y \in K$. The answer is "no". The following example is given in [21]:

Let $K=[0,1] \subset \mathbb{R}$. Let $\varphi:\left[\frac{1}{4}, \frac{1}{2}\right) \rightarrow\left[\frac{3}{4}, 1\right]$ be an arbitrary one-to-one surjective function and define $T:[0,1] \rightarrow[0,1]$ by setting

$$
T x= \begin{cases}x+\frac{1}{2} & \text { for } x \in\left[0, \frac{1}{4}\right) \\ \varphi(x) & \text { for } x \in\left[\frac{1}{4}, \frac{1}{2}\right) \\ x-\frac{1}{2} & \text { for } x \in\left[\frac{1}{2}, \frac{3}{4}\right) \\ \varphi^{-1}(x) & \text { for } x \in\left[\frac{3}{4}, 1\right]\end{cases}
$$

This function is obviously discontinuous. However, $T^{2} x=x$ for all $x \in[0,1]$ so $T$ satisfies (8). On the other hand, $|x-T x| \geq \frac{1}{4}$ for all $x \in[0,1]$, so $T$ fails to have approximate fixed points.

This example is a little surprising in view of the fact that the slightly stronger condition

$$
\min \left\{\rho(T x, T y), \rho\left(T^{2} x, T^{2} y\right)\right\} \leq k \rho(x, y)
$$

for all $x, y \in M$ actually implies that $T$ has a fixed point in any complete metric space. Condition (9) is just the Generalized Banach Contraction Conjecture (GBBC) of [32] for the case $n=2$. (The GBBC was first fully solved in [43]. For more on the GBBC we refer to the discussion in [19].) Such mappings always have unique fixed points despite the fact that they may be discontinuous. Also, in addition to (9), if $T$ is uniformly continuous, then convergence of the Picard iterates follows from a recent result of Reich and Zaslavski [48].

In looking to strengthen (8) to obtain a mapping with better properties, one might immediately think of the condition

$$
\max \left\{\|T x-T y\|,\left\|T^{2} x-T^{2} y\right\|\right\} \leq\|x-y\|
$$

for each $x, y \in K$. However, this is just another way of saying $T$ is nonexpansive. On the other hand, there are conditions that are formally between (8) and (10). The simplest which might come to mind is

$$
\frac{\|T x-T y\|+\left\|T^{2} x-T^{2} y\right\|}{2} \leq\|x-y\|
$$

for each $x, y \in K$. This observation motivated the further study in [21].

Condition (11) immediately implies that both the mappings $T$ and $T^{2}$ are 2-lipschitzian. In fact, applying (11) to points $T x$ and $T y$ and adding $2\left\|T^{2} x-T^{2} y\right\|$ to both sides of the inequality, we obtain

$$
3\left\|T^{2} x-T^{2} y\right\|+\left\|T^{3} x-T^{3} y\right\| \leq 2\left(\|T x-T y\|+\left\|T^{2} x-T^{2} y\right\|\right) \leq 4\|x-y\|,
$$

and this implies that $T^{2}$ is $\frac{4}{3}$-lipschitzian.

Does a mapping satisfying Condition (11) have approximate fixed points? The answer now is "yes". Let $F:=\frac{T+T^{2}}{2}$. Then for all $x, y \in K$,

$$
\begin{aligned}
\|F x-F y\| & =\frac{\left\|T x+T^{2} x-\left(T y+T^{2} y\right)\right\|}{2} \\
& \leq \frac{\|T x-T y\|+\left\|T^{2} x-T^{2} y\right\|}{2} \\
& \leq\|x-y\| .
\end{aligned}
$$


Therefore, $F$ is nonexpansive, so given $\varepsilon>0$ there exists $x_{\varepsilon} \in K$ such that $\left\|x_{\varepsilon}-F x_{\varepsilon}\right\|<\varepsilon$. Now, putting $y=T x_{\varepsilon}$ in (11), we have

$$
\begin{aligned}
\frac{\left\|T x_{\varepsilon}-T^{2} x_{\varepsilon}\right\|+\left\|T^{2} x_{\varepsilon}-T^{3} x_{\varepsilon}\right\|}{2} & \leq\left\|x_{\varepsilon}-T x_{\varepsilon}\right\| \\
& \leq\left\|x_{\varepsilon}-F x_{\varepsilon}\right\|+\left\|F x_{\varepsilon}-T x_{\varepsilon}\right\| \\
& <\left\|F x_{\varepsilon}-T x_{\varepsilon}\right\|+\varepsilon \\
& =\left\|\frac{T x_{\varepsilon}+T^{2} x_{\varepsilon}}{2}-T x_{\varepsilon}\right\|+\varepsilon \\
& \leq\left\|\frac{T^{2} x_{\varepsilon}-T x_{\varepsilon}}{2}\right\|+\varepsilon .
\end{aligned}
$$

This implies $\left\|T^{2} x_{\varepsilon}-T^{3} x_{\varepsilon}\right\|<2 \varepsilon$, and since $\varepsilon>0$ is arbitrary

$$
\inf \{\|x-T x\|: x \in K\}=0 .
$$

Returning to Condition (11), if a closed convex subset $K$ of a Banach space has the fixed point property for mappings satisfying this condition, then it must also have the fixed point property for nonexpansive mappings. So we suppose $K$ has the fpp, and suppose $T: K \rightarrow K$ satisfies (11). Then the mapping $F:=\frac{T+T^{2}}{2}$ has at least one fixed point, say $x_{0}$. Putting $y=T x_{0}$ in (11) we have

$$
\frac{\left\|T x_{0}-T^{2} x_{0}\right\|+\left\|T^{2} x_{0}-T^{3} x_{0}\right\|}{2} \leq\left\|x_{0}-T x_{0}\right\|=\left\|\frac{T^{2} x_{0}-T x_{0}}{2}\right\| .
$$

Consequently, $\left\|T^{2} x_{0}-T^{3} x_{0}\right\| \leq 0$, so $T^{2} x_{0}$ is a fixed point of $T$. Thus we have the following:

Theorem 5.1 If a convex subset of a Banach space $K$ has the fixed point property for nonexpansive mappings, then it has the fixed point property for mappings satisfying (11).

Indeed, we have the following situation: $T^{2}(f i x(F)) \subseteq f i x(T) \subseteq f i x(F)$.

Question 1 Are there mappings for which the above inclusions are strict?

The answer to this question is "no" if the Lipschitz constant, $k(T)$, of $T$ is strictly less than 2.

Theorem 5.2 Suppose a convex subset of a Banach space $K$ has the fixed point property for nonexpansive mappings, and suppose $T: K \rightarrow K$ satisfies $(i) F:=\frac{1}{2}\left(T+T^{2}\right)$ is nonexpansive and (ii) $k(T)<2$. Then $f i x(T)=f i x(F) \neq \emptyset$.

Proof If $F(x)=x$ then $\left\|T x-T^{2} x\right\|=2\|x-T x\|$. Since $k(T)<2$, it must be the case that $x=T x$.

Condition (i) alone in the above theorem implies neither the continuity of $T$ nor the existence of a fixed point. The simplest example is the following. Define $T:[0,1] \rightarrow[0,1]$ as follows:

$$
T x= \begin{cases}1 & \text { for } x \in\left[0, \frac{1}{2}\right] \\ 0 & \text { for } x \in\left(\frac{1}{2}, 1\right] .\end{cases}
$$

Then

$$
T^{2} x= \begin{cases}0 & \text { for } x \in\left[0, \frac{1}{2}\right] \\ 1 & \text { for } x \in\left(\frac{1}{2}, 1\right]\end{cases}
$$

so

$$
F x=\frac{T x+T^{2} x}{2} \equiv \frac{1}{2}
$$

The preceding simple discussion gives rise to several questions. While the mean (11) is elegant, one could also consider weighted averages: 
Question 2 Can Condition (11) in Theorem 5.1 be replaced by the assumption that for some $\alpha \in(0,1) T$ satisfies

$$
\alpha\|T x-T y\|+(1-\alpha)\left\|T^{2} x-T^{2} y\right\| \leq\|x-y\|
$$

for each $x, y \in K$ ?

An argument similar to the one given above shows that the answer is affirmative if $\alpha \geq \frac{1}{2}$. In fact this is a special case of the following more general result. For $n \geq 2$, let $\alpha=\left(\alpha_{1}, \alpha_{2}, \ldots, \alpha_{n}\right)$ be a multiindex satisfying

$$
\alpha_{1}>0, \alpha_{n}>0, \alpha_{i} \geq 0, i=1,2, \ldots, n, \text { and } \sum_{i=1}^{n} \alpha_{i}=1 .
$$

A mapping $T: K \rightarrow K$ is called $\alpha$-nonexpansive if

$$
\sum_{i=1}^{n} \alpha_{i}\left\|T^{i} x-T^{i} x\right\| \leq\|x-y\|
$$

for each $x, y \in K$. It can be shown that this condition implies that the mapping

$$
T_{\alpha} x:=\alpha_{1} T x+\alpha_{2} T^{2} x+\cdots+\alpha_{n} T^{n} x
$$

for $x \in K$ is nonexpansive, and a further technical argument yields the following:

Theorem 5.3 [21] If a convex subset of a Banach space $K$ has the fixed point property for nonexpansive mappings, then it has the fixed point property for $\alpha$-nonexpansive mappings when $\alpha_{1} \geq \frac{1}{2^{\frac{1}{n-1}}}$.

The justification of the fact that the above result is a kind of stability comes from the following two observations:

First, following [28], put

$$
d(x, y)=\sum_{j=1}^{n}\left(\sum_{i=j}^{n} \alpha_{i}\right)\left\|T^{j-1} x-T^{j-1} y\right\|
$$

and notice that $d(x, y)$ is a metric equivalent to the one defined by the norm. Now, (12) means exactly that

$$
d(T x, T y) \leq d(x, y) .
$$

Hence all nonexpansive mappings, as nonexpansive with respect to equivalent metric, are uniformly lipschitzian. Thus the fixed point property for nonexpansive mappings is stable with respect to special changes of metrics. However, it was also observed in [28] that there are uniformly lipschitzian mappings which are not $\alpha$-nonexpansive for any $\alpha$.

The second observation comes from the very recent Ph.D. thesis of Piasecki [45] (see also [46]). Obviously, for any $\alpha$ the class of $\alpha$ - nonexpansive mappings contain all nonexpansive ones. However, on any convex set of positive diameter and for any $\alpha$ there are mappings which are $\alpha$-nonexpansive but not nonexpansive. Some of them can even satisfy $k\left(T^{n}\right)>1$ for $n=1,2,3, \ldots$. Thus the notion of mean nonexpansiveness is not only a formal but also a proper generalization of nonexpansiveness

The estimate $\alpha_{1} \geq \frac{1}{2^{\frac{1}{n-1}}}$ from the last theorem does not give complete information about all $\alpha$-nonexpansive mappings having fixed points. Even in case $n=2$ it is not known if the condition $\alpha_{1} \geq \frac{1}{2}$ is the best possible. Also, it was observed in [21] that in case $n=3$, if we consider only monotone $\alpha=\left(\alpha_{1}, \alpha_{2}, \alpha_{3}\right), \alpha_{1} \geq \alpha_{2} \geq \alpha_{3}$, the sufficient condition can be relaxed from $\alpha_{1} \geq \frac{1}{\sqrt{2}}$ to $\alpha_{1} \geq \frac{1}{2}$.

Question 3 Given $n=2,3,4, \ldots$, is it possible to describe completely, or at least give partial information, about the set $A_{n}$ consisting of all $\alpha^{\prime} s$ of length $n$, such that fpp for nonexpansive mappings implies fpp for $\alpha$ -nonexpansive ones? 


\section{Uniformly lipschitzian mappings in geodesic spaces}

It is possible to at least partially extend the results described in the previous two sections to certain geodesic spaces. A metric space $(X, \rho)$ is a geodesic space if each two points $p, q \in X$ can be joined by a geodesic, that is, by a path $\gamma:[a, b] \rightarrow X$ where $[a, b]$ is a real line interval, $\gamma$ is an isometry, and $\gamma(a)=p, \gamma(b)=q$.

We first consider a special class of geodesic spaces called the CAT $(\kappa)$ spaces, and we consider only the case $\kappa \leq 0$. A geodesic space $(X, d)$ is said to be a $C A T(\kappa)$ space (the term is due to M. Gromov- see, e.g., [4], p. 159) if it is geodesically connected and has constant curvature bounded above by $\kappa$. More precisely, every geodesic triangle in $X$ is at least as 'thin' as its comparison triangle in $M_{\kappa}^{2}$, where for $\kappa<0 M_{\kappa}^{2}$ is the real hyperbolic space $\mathbb{H}^{2}$ with the distance function scaled by a factor of $1 / \sqrt{-\kappa}$, and if $\kappa=0, M_{\kappa}^{2}$ is the Euclidean plane. For precise definitions and a thorough discussion of these spaces and of the fundamental role they play in various branches of mathematics, see Bridson and Haefliger [4] or Burago et al. [10]. We note in particular that the complex Hilbert ball with a hyperbolic metric (see [29]; also inequality (4.3) of [47] and subsequent comments) is a CAT( 0$)$ space.

There are interesting spaces which are $\operatorname{CAT}(\kappa)$ for all $\kappa \leq 0$.

Definition 6.1 An $\mathbb{R}$-tree (or metric tree) is a metric space $M$ such that

(i) there is a unique geodesic (metric) segment denoted by $[x, y]$ joining each pair of points $x$ and $y$ in $M$; and

(ii) $[y, x] \cap[x, z]=\{x\} \Rightarrow[y, x] \cup[x, z]=[y, z]$.

In [12], it was proved that the Lifšic constant $\kappa(X)$ for any CAT $(\kappa)$ space $X$ with $\kappa \leq 0$ satisfies $\kappa(X) \geq$ $\sqrt{2}$, and $\kappa(X)=2$ if $X$ is an $\mathbb{R}$-tree. It was conjectured there that the Lifšic constant of a CAT $(\kappa)$ space for $\kappa<0$ is a continuous decreasing function of $\kappa$ which takes values in the interval $(\sqrt{2}, 2)$. The authors have informed us that an affirmative answer to this conjecture given in [15] is only partially correct.

In view of these observations and Lifšic's theorem (and Remark 3.5), we have the following:

Theorem 6.2 Let $X$ complete CAT(0) space and suppose $T: K \rightarrow K$ has bounded orbits and satisfies

$$
d\left(T^{n} x, T^{n} y\right) \leq k_{n} d(x, y)
$$

for each $x, y \in K$, where $\lim \sup _{n \rightarrow \infty} k_{n}<\sqrt{2}$. Then $T$ has a fixed point.

For an $\mathbb{R}$-tree we have the following result due to Aksoy and Khamsi [1].

Theorem 6.3 Let $X$ be a complete $\mathbb{R}$-tree, and suppose $T: X \rightarrow X$ has bounded orbits, and suppose there exists $n_{0}$ such that for $n \geq n_{0} T$ and satisfies

$$
d\left(T^{n} x, T^{n} y\right) \leq k_{n} d(x, y)
$$

for each $x, y \in X$, where $\lim \sup _{n \rightarrow \infty} k_{n}<2$. Then $T$ has a fixed point.

\section{Mean nonexpansive mappings in geodesic spaces}

We now turn to a more general class of geodesic spaces. A Busemann space (we adopt the terminology of [44]) is a geodesic metric space $X$ such that for any two geodesics $\gamma:[a, b] \rightarrow X$ and $\gamma^{\prime}:\left[a^{\prime}, b^{\prime}\right] \rightarrow X$, the map from $D_{\gamma, \gamma^{\prime}}:[a, b] \times\left[a^{\prime}, b^{\prime}\right] \rightarrow \mathbb{R}$ defined by

$$
D_{\gamma, \gamma^{\prime}}\left(t, t^{\prime}\right) \rightarrow d\left(\gamma(t), \gamma^{\prime}\left(t^{\prime}\right)\right)
$$

is convex. Equivalently, let $\left[x_{0}, x_{1}\right]$ and $\left[x_{0}^{\prime}, x_{1}^{\prime}\right]$ be two geodesic segments in $X$. For every $t \in[0,1]$ let $x_{t}$ be the point on $\left[x_{0}, x_{1}\right]$ satisfying $d\left(x_{0}, x_{t}\right)=t d\left(x_{0}, x_{1}\right)$ and let $x_{t}^{\prime}$ be the point on $\left[x_{0}^{\prime}, x_{1}^{\prime}\right]$ satisfying $d\left(x_{0}^{\prime}, x_{t}^{\prime}\right)=t d\left(x_{0}^{\prime}, x_{1}^{\prime}\right)$. Then,

$$
d\left(x_{t}, x_{t}^{\prime}\right) \leq(1-t) d\left(x_{0}, x_{0}^{\prime}\right)-t d\left(x_{1}, x_{1}^{\prime}\right) .
$$

The following two conditions are necessary and sufficient conditions for a geodesic metric space $X$ to be a Busemann space. The second follow immediately from the first by two applications of the triangle inequality. 

Then

(i) Let $\left[x_{0}, x_{1}\right]$ and $\left[x_{0}^{\prime}, x_{1}^{\prime}\right]$ be two geodesic segments in $X$, and let $m$ and $m^{\prime}$ be there respective midpoints.

$$
d\left(m, m^{\prime}\right) \leq \frac{1}{2}\left(d\left(x_{0}, x_{1}\right)+d\left(x_{0}^{\prime}, x_{1}^{\prime}\right)\right) .
$$

In a Busemann space the geodesic joining any two points is unique. To see this let $\left[x_{0}, x_{1}\right]$ and $\left[x_{0}^{\prime}, x_{1}^{\prime}\right]$ be two geodesic segments in $X$. For every $t \in[0,1]$ let $x_{t}$ be the point on $\left[x_{0}, x_{1}\right]$ satisfying $d\left(x_{0}, x_{t}\right)=t d\left(x_{0}, x_{1}\right)$ and let $x_{t}^{\prime}$ be the point on $\left[x_{0}^{\prime}, x_{1}^{\prime}\right]$ satisfying $d\left(x_{0}^{\prime}, x_{t}^{\prime}\right)=t d\left(x_{0}^{\prime}, x_{1}^{\prime}\right)$. Then

$$
d\left(x_{t}, x_{t}^{\prime}\right) \leq(1-t) d\left(x_{0}, x_{0}^{\prime}\right)-t d\left(x_{1}, x_{1}^{\prime}\right) .
$$

From this we see that if $x_{0}=x_{0}^{\prime}$ and $x_{1}=x_{1}^{\prime}$ then it follows that $x_{t}=x_{t}^{\prime}$ for all $t \in[0,1]$. Thus these spaces are precisely spaces which are said to be of hyperbolic type in the terminology of [23].

The key observation to extending the results of Sect. 5 to geodesic spaces is the Busemann space inequality (14). It is known that bounded closed convex subsets of complete CAT( 0 ) spaces have the fixed point property for nonexpansive mappings, as do bounded hyperconvex metric space spaces. These spaces are also Busemann spaces. As a result, we immediately have the following extension of Theorem 5.1.

Theorem 7.1 If $(X, \rho)$ is a Busemann convex space which has the fixed point property for nonexpansive mappings, then $X$ has the fixed point property for mappings $T: X \rightarrow X$ satisfying

$$
\frac{\rho(T x, T y)+\rho\left(T^{2} x, T^{2} y\right)}{2} \leq \rho(x, y)
$$

for each $x, y \in X$.

Proof For each $x \in X$, let $F x$ denote the unique midpoint of the geodesic joining $T x$ and $T^{2} x$. It is immediate from (14) that $F$ is nonexpansive. Letting $x_{0}$ denote a fixed point of $F$, and putting $y=T x_{0}$ in (15), we have

$$
\frac{\rho\left(T x_{0}, T^{2} x_{0}\right)+\rho\left(T^{2} x_{0}, T^{3} x_{0}\right)}{2} \leq \rho\left(x_{0}, T x_{0}\right)=\frac{\rho\left(T x_{0}, T^{2} x_{0}\right)}{2}
$$

from which $\rho\left(T^{2} x_{0}, T^{3} x_{0}\right)=0$.

The question might naturally arise whether there is an analog of Theorem 5.3 for Busemann spaces. The answer to this is not so clear because the definition of higher order convex combinations of points in Busemann spaces is not as straight forward as in normed linear spaces. We propose to take up this matter in more detail elsewhere.

\section{Uniform normal structure}

Let $(M, d)$ be a metric space. A nonempty subset $A$ of $M$ is said to be admissible if $A$ is an intersection of closed balls of $M$. Let $\mathcal{A}(M)$ denote the collection of all nonempty admissible subsets of $M$. Thus $A \in \mathcal{A}(M) \Leftrightarrow A \neq \emptyset$ and

$$
A=\cap_{i \in I} B\left(x_{i} ; r_{i}\right)
$$

where $x_{i} \in M, r_{i} \geq 0$, and $i \in I$ for some index set $I$.

For $D \in \mathcal{A}(M)$, let

$$
r(D)=\inf \{\rho>0: D \subseteq B(x ; \rho) \text { for some } x \in M\}
$$

and let

$$
\mathcal{C}(D)=\{x \in M: D \subseteq B(x ; r(D))\} .
$$

The number $r(D)$ is called the Chebyshev radius of $D$, and $\mathcal{C}(D)$ is called the Chebyshev center of $D$. The family $\mathcal{A}(M)$ is said to be normal if $r(D)<\operatorname{diam}(D)$ whenever diam $(D)>0$, and $\mathcal{A}(M)$ is said to be compact if every descending chain of nonempty subsets of $\mathcal{A}(M)$ has nonempty intersection. $\mathcal{A}(M)$ is said to be countably compact if every descending sequence of nonempty subsets of $\mathcal{A}(M)$ has nonempty intersection.

We first observe that if $\mathcal{A}(M)$ is compact, then $\mathcal{C}(D) \neq \emptyset$ for each nonempty set $D$ in $\mathcal{A}(M)$.

The following is an abstract version of the original theorem of [34]. 
Theorem 8.1 Let $(M, \rho)$ be a metric space for which the admissible sets are countably compact and normal. Then $M$ has the fpp for nonexpansive mappings.

Suppose $(M, d)$ is a metric space. The admissible sets $\mathcal{A}(X)$ of a metric space $(X, d)$ are said to be uniformly normal with constant $c<1$ if for any $D \in \mathcal{A}(X)$ with $\operatorname{diam}(D)>0, r(D) / \operatorname{diam}(D) \leq c$. In a Banach space, uniform normal structure is known to imply reflexivity, see [41]. Also, in a uniformly convex Banach space, the admissible sets are uniformly normal in the sense described here.

The following example shows that uniform normal structure completely unstable under small perturbations of the original metric. (This example corrects a slight flaw in the example described in [36].) Therefore, despite the fact that Banach spaces for which $\varepsilon_{0}(X)<1$ have uniform normal structure, it seems unlikely that one could obtain results similar to those of Casini and Maluta [11] in this more abstract setting. However, the question remains open.

Example We remetrize the unit ball $X$ in $\mathbb{R}_{\infty}^{2}$. Let $p=(1,0) ; q=(-1,0) ; u=(0,-1) ; v=(0,1)$ in $\mathbb{R}_{\infty}^{2}$. Let 0 denote the origin and let $\varepsilon>0$. Let $0^{\prime}$ be a point in $\mathbb{R}^{3}$ on a line passing through 0 and perpendicular to the plane $\mathbb{R}^{2}$ such that the (euclidean) distance between 0 and $0^{\prime}$ is $\varepsilon$. Let $X^{\prime}$ be the surface in $\mathbb{R}^{3}$ formed by taking the union of the segments $\left[w, 0^{\prime}\right]$ where $w$ is on the boundary of $X$. For each $x \in X$, let $x^{\prime}$ be the point of $X^{\prime}$ which is directly above $X$, and let $\ell(x)$ denote the length of the segment $\left[x, x^{\prime}\right]$. Now assign a new metric $\rho$ to $X$ by taking

$$
\rho(x, y)=\left(\left(d_{\infty}(x, y)\right)^{2}+|\ell(x)-\ell(y)|^{2}\right)^{\frac{1}{2}} .
$$

Clearly $\rho$ is a metric on $X$. Also for any $x, y \in X$,

$$
d_{\infty}(x, y) \leq \rho(x, y) \leq k d_{\infty}(x, y)
$$

where $k=\sqrt{1+\varepsilon^{2}}$. However, since $\rho=d_{\infty}$ on the boundary of $X$,

$$
\rho(p, v)=\rho(q, v)=\rho(p, u)=\rho(q, u)=1 .
$$

On the other hand, $\rho(p, w)=\rho(q, w)=\left(1+(\ell(w))^{2}\right)^{\frac{1}{2}}>1$ for any point $w$ in the open segment $(u, v)$. Therefore, $B_{\rho}(p ; 1) \cap B_{\rho}(q ; 1)=\{u, v\}$, so $B_{\rho}(p ; 1) \cap B_{\rho}(q ; 1)$ is an example of an admissible set whose intersection consists of precisely two diametral points. Thus, not only does $(X, \rho)$ not have uniform normal structure; it does not even have normal structure. We also note that $X$ has uniform normal structure with constant $1 / 2$ (the best possible!) because $\mathbb{R}_{\infty}^{2}$ is hyperconvex.

Acknowledgments The research of K. Bolibok was partially supported by the Ministry of Science and Higher Education, Poland, N N201 393737.

Open Access This article is distributed under the terms of the Creative Commons Attribution License which permits any use, distribution, and reproduction in any medium, provided the original author(s) and the source are credited.

\section{References}

1. Aksoy, A.G.; Khamsi, M.A.: Fixed points of uniformly lipschitzian mappings in metric trees. Sci. Math. Jpn. 65, 31-41 (2007); e:2006, 1143-1153

2. Baillon, J.-B.: Un théorème de type ergodique pour les contractions non linéaires dans un espace de Hilbert (French). C. R. Acad. Sci. Paris Sér. A-B 280(22), Aii, A1511-A1514 (1975)

3. Belluce, L.P.; Kirk, W.A.; Steiner, E.F.: Normal structure in Banach spaces. Pac. J. Math. 26, $433-440$ (1968)

4. Bridson, M.; Haefliger, A.: Metric Spaces of Non-Positive Curvature. Springer, Berlin (1999)

5. Brodskii, M.S.; Milman, D.P.: On the center of a convex set (Russian). Dokl. Akad. Nauk. SSSR 59, $837-840$ (1948)

6. Browder, F.E.: Nonexpansive nonlinear operators in a Banach space. Proc. Natl. Acad. Sci. 54, 1041-1044 (1965)

7. Bruck, R.E.: Properties of fixed-point sets of nonexpansive mappings in Banach spaces. Trans. Am. Math. Soc. 179, 251-262 (1973)

8. Bruck, R.E.: A common fixed point theorem for a commuting family of nonexpansive mappings. Pac. J. Math. 53, 59-71 (1974)

9. Bruck, R.E.: Structure of approximate fixed point sets of nonexpansive mappings in general Banach spaces. Fixed point theory and applications (Marseille, 1989). Pitman Research Notes in Mathematics Series, vol. 252, pp. 91-96. Longman Scientific and Techhical, Harlow (1991) 
10. Burago, D.; Burago, Y.; Ivanov, S.: A Course in Metric Geometry. Graduate Studies in Mathematics, vol. 33. American Mathematical Society, Providence (2001)

11. Casini, E.; Maluta, E.: Fixed points of uniformly Lipschitzian mappings in spaces with uniformly normal structure. Nonlinear Anal. 9(1), 103-108 (1985)

12. Dhompongsa, S.; Kirk, W.A.; Sims, B.: Fixed points of uniformly Lipschitzian mappings. Nonlinear Anal. 65(4), 762-772 (2006)

13. Dowling, P.N.; Lennard, C.J.; Turett, B.: Some fixed point results in $l^{1}$ and $c_{0}$. Nonlinear Anal. Ser. A Theory Methods 39(7), 929-936 (2000)

14. Downing, D.J.; Turett, B.: Some properties of the characteristic of convexity relating to fixed point theory. Pac. J. Math. 104, 343-350 (1983)

15. Espínola, R.; Fernández-León, A.: CAT( $\kappa)$-spaces, weak convergence and fixed points. J. Math. Anal. Appl. 353(1), 410-427 (2009)

16. Espínola, R.; Khamsi, M.A.: Introduction to hyperconvex spaces. Handbook of Metric Fixed Point Theory, pp. $391-435$. Kluwer, Dordrecht (2001)

17. Garcia-Falset, J.; Jiménez-Melado, A.; Llorens-Fuster, E.: Stability of the fixed point property for nonexpansive mappings. Handbook of Metric Fixed Point Theory, pp. 201-238. Kluwer, Dordrecht (2001)

18. Goebel, K.: Convexity of balls and fixed point theorems for mappings with nonexpansive square. Compositio Math. 22, 269-274 (1970)

19. Goebel, K.: Concise Course on Fixed Point Theorems. Yokohama Publishers, Yokohama (2002)

20. Goebel, K.: Examples for an old problem on commuting mappings. Fixed Point Theory and Its Applications, pp. 71-75. Yokohama Publishers, Yokohama (2010)

21. Goebel, K.; Japón Pineda, M.A.: On a type of generalized nonexpansiveness. Fixed Point Theory and Its Applications, pp. 71-82. Yokohama Publishers, Yokohama (2008)

22. Goebel, K.; Kirk, W.A.: A fixed point theorem for transformations whose iterates have uniform Lipschitz constant. Studia Math. 47, 135-140 (1973)

23. Goebel, K.; Kirk, W.A.: Iteration processes for nonexpansive mappings, Topological methods in nonlinear functional analysis (Toronto, Ont., 1982). Contemporary Mathematics, vol. 21, pp. 115-123. American Mathematical Society, Providence (1983)

24. Goebel, K.; Kirk, W. A.: Topics in metric fixed point theory. Cambridge Studies in Advanced Mathematics, vol. 28. Cambridge University Press, Cambridge (1990)

25. Goebel, K.; Kirk, W.A.: Classical theory of nonexpansive mappings. Handbook of Metric Fixed Point Theory, pp. 49-91. Kluwer, Dordrecht (2001)

26. Goebel, K.; Kirk, W.A.: Some problems in metric fixed point theory. J. Fixed Point Theory Appl. 4(1), 13-25 (2008)

27. Goebel, K.; Kirk, W.A.; Thele, R.L.: Uniformly lipschitzian families of transformations in Banach spaces. Can. J. Math. 31, $1245-1256(1974)$

28. Goebel, K.; Sims, B.: Mean Lipschitzian mappings. Nonlinear Analysis and Optimisation I, Nonlinear Analysis. Contemporary Mathematics, vol. 513, pp. 157-167 (2010)

29. Goebel, K.; Reich, S.: Uniform Convexity, Hyperbolic Geometry, and Nonexpansive Mappings. Marcel Dekker, Inc., New York (1984)

30. Göhde, D.: Zum Prinzip der kontraktiven Abbildung. Math. Nachr. 30, 251-258 (1965)

31. Hernández, C.; Japón, M.; Llorens-Fuster, E.: On the structure of the set of equivalent norms on $\ell_{1}$ with the fixed point property. J. Math. Anal. Appl. 387(2), 645-654 (2012)

32. Jachymski, J.R.; Schröder, B.; Stein, J.D. Jr.: A connection between fixed-point theorems and tiling problems. J. Combin. Theory Ser. A 87, 273-286 (1999)

33. Khamsi, M.A.; Kirk, W.A.: On uniformly lipschitzian multivalued mappings in Banach and metric spaces. Nonlinear Anal. 72, 2080-2085 (2010)

34. Kirk, W.A.: A fixed point theorem for mappings which do not increase distance. Am. Math. Monthly 72, 1002-1004 (1965)

35. Kirk, W.A.; Sims, B. (eds.): Handbook of Metric Fixed Point Theory. Kluwer, Dordrecht (2001)

36. Kirk, W.A.: The approximate fixed point property and uniform normal structure in hyperconvex spaces. In: Proceedings of International Conference on Nonlinear and Convex Analysis (Hirosaki, 2001), pp. 179-190. Yokohama Publishers, Yokohama (2003)

37. Kirk, W.A.: Fixed point theorems in CAT(0) spaces and R-trees. Fixed Point Theory Appl. 2004(4), 309-316 (2004)

38. Lifšic, E.A.: Fixed point theorems for operators in strongly convex spaces, Voronež Gos. Univ. Trudy Mat. Fak., 16, 23-28 (1975) (Russian)

39. Lin, P.-K.: Stability of the fixed point property of Hilbert spaces. Proc. Am. Math. Soc. 127(12), 3573-3581 (1999)

40. Lin, P.-K.: There is an equivalent norm on $\ell^{1}$ that has the fixed point property. Nonlinear Anal. 68(8), 2303-2308 (2008)

41. Maluta, E.: Uniformly normal structure and related coefficients. Pac. J. Math. 111, 357-369 (1984)

42. Mazcuñán-Navarro, E.: Stability of the fixed point property in Hilbert spaces. Proc. Am. Math. Soc. 134(1), 129-138 (2006)

43. Merryfield, J.; Stein, J.D. Jr.: A generalization of the Banach contraction principle. J. Math. Anal. Appl. 273, 112-120 (2002)

44. Papadopoulos, A.: Metric spaces, convexity and nonpositive curvature. IRMA Lectures in Mathematics and Theoretical Physics, vol. 6, xii+287 pp. European Mathematical Society (EMS), Zürich (2005)

45. Piasecki, Ł.: On a classification of Lipschitz mappings (in Polish). PhD thesis, Maria Curie-Sklodowska University, Lublin, Poland (2012)

46. Perez-Garcia, V.; Piasecki, Ł.: Lipschitz constants for iterations of mean lipschitzian mappings. Nonlinear Anal. 74(16), 5643-5647 (2011)

47. Reich, S.; Shafrir, I.: Nonexpansive iterations in hyperbolic spaces. Nonlinear Anal. 15, 537-558 (1990)

48. Reich, S.; Zaslavski, A.J.: Two results on Jachymski-Schrö der-Stein contractions. Bull. Polish Acad. Sci. Math. 56, 53-58 (2008) 